\title{
Clinical application of fractional exhaled nitric oxide and nasal nitric oxide levels for the assess eosinophilic inflammation of allergic rhinitis among children
}

\author{
Jia-Ying Luo", Hui-An Chen", Jing Ma, Yong-Xin Xiao, Jing-Jiong Yao, Jia-Min Liang, Ying-Si Du, \\ Feng Wang, Bao-Qing Sun
}

Department of Allergy and Clinical Immunology, State Key Laboratory of Respiratory Disease, National Clinical Research Center of Respiratory Disease, Guangzhou Institute of Respiratory Health, First Affiliated Hospital of Guangzhou Medical University, Guangzhou Medical University, Guangzhou, China

Contributions: (I) Conception and design: JY Luo; (II) Administrative support: BQ Sun; (III) Provision of study materials or patients: F Wang; (IV) Collection and assembly of data: HA Chen, J Ma, YX Xiao, JJ Yao, YS Du; (V) Data analysis and interpretation: JY Luo, JM Liang; (VI) Manuscript writing: All authors; (VII) Final approval of manuscript: All authors.

\#These authors contributed equally to this work.

Correspondence to: Feng Wang; Bao-Qing Sun. State Key Laboratory of Respiratory Disease, First Affiliated Hospital of Guangzhou Medical University, 151 Yanjiangxi Road, Guangzhou 510120, China. Email: 412475734@qq.com; sunbaoqing@vip.163.com.

Background: Allergic rhinitis is one of the most common nasal inflammatory diseases among children. Assessment of clinical symptoms, skin prick test and serum immunoglobulin E ( $\operatorname{IgE}$ ) are common methods used to diagnose allergic rhinitis and assess inflammation degree in clinical settings. However, via blood tests assess eosinophils inflammation is invasive, and may cause fear in children. It makes have burden of the diagnosis of allergic rhinitis. Nasal nitric oxide (nNO) and fractional exhaled nitric oxide (FeNO) are noninvasive, inexpensive, and can provide immediate results. These methods may therefore be preferable to assess the inflammation of allergic rhinitis.

Methods: This study was a retrospective analysis. We recruited 61 children with allergic rhinitis from November 2019 to March 2020. The participants were assessed using the FeNO and nNO tests. We also administered questionnaires and carried out traditional allergen and blood tests. We analyzed the relationship between diagnosis results and $\mathrm{FeNO}$ and $\mathrm{nNO}$ levels before and after the treatment of allergic rhinitis, to investigate the clinical application of $\mathrm{FeNO}$ and $\mathrm{nNO}$ levels for assess eosinophilic inflammation of allergic rhinitis in children.

Results: We observed a significant association both FeNO, nNO level with eosinophils, total IgE. In different levels of eosinophils (EOS), the correlation of detection parameters had obvious change. FeNO and nNO levels were obvious higher compared to pre-treatment.

Conclusions: Using NO concentration can indicates the extent of allergic inflammation and can measure allergy treatment effects combine other influence indexes. The combined use of FeNO and nNO levels may be a useful method for assess the degree of eosinophilic inflammation of allergic rhinitis in children.

Keywords: Allergic rhinitis; fractional exhaled nitric oxide (FeNO); nasal nitric oxide (nNO); children; eosinophils

Submitted Sep 30, 2020. Accepted for publication Jan 29, 2021.

doi: $10.21037 /$ tp-20-314

View this article at: http://dx.doi.org/10.21037/tp-20-314 


\section{Introduction}

Allergic rhinitis is a global health problem and affects people of all ages. Moreover, the prevalence of this condition has increased annually. Approximately 40\% of children present with allergic rhinitis worldwide (1). In China, the proportion of individuals with allergic rhinitis increased from $11.1 \%$ to $17.7 \%$ within the last six years (2). Questionnaires are widely used to assess the prevalence of allergic rhinitis. However, it they may overestimate the prevalence of allergic rhinitis (3). The morbidity associated with allergic rhinitis is significantly higher in major cities. For example, in Beijing and Shanghai the percentages of people with allergic rhinitis were $20.2 \%$ and $23.0 \%$ in 2011, respectively (2). Due to worsening air pollution, and changes in lifestyle habits, the morbidity of nasal inflammatory diseases has been trending upward (4).

Allergic rhinitis is an inflammatory response caused by immunoglobulin $\mathrm{E}$ ( $\mathrm{IgE}$ )-mediated sensitivity to environmental allergens (5). Nasally inhaled sensitizing allergens promote the synthesis of $\mathrm{IgE}$ and strongly activate the activation of mast cells, eosinophils (EOS), and T-helper type 2 cells. Approximately $19-38 \%$ of individuals with allergic rhinitis have concurrent asthma (6). The main clinical symptoms include a runny nose, rhinorrhea, nasal congestion, and itching of the nose and eyes. Depending on symptom severity, allergic rhinitis can exert similarly severe effects on patients' daily lives and sleep habits.

Traditional methods of assessing the clinical symptoms of allergic rhinitis include the skin prick test and detection of serum allergens (7). A visual analog scale can be used to effectively assess the symptom severity (8); however, some study point out that allergen test is invasive and may incite fear in children. Further, the high cost and long inspection time increase the burden of these tests (9). Moreover, allergic rhinitis can be difficult to distinguish from other common diseases among young children (10).

Measurement of nitric oxide (NO) concentrations in exhaled breath is a useful method for detecting the severity of airway inflammation. $\mathrm{NO}$ is generated via three $\mathrm{NO}$ synthases and is expressed in epithelial and inflammatory cells (10). In the respiratory system, NO acts as a messenger to transform intercellular information and regulate the dilation of the bronchus and blood vessels. Fractional exhaled nitric oxide (FeNO) and nasal nitric oxide (nNO) levels correspond with inflammation in the lower and upper airways and eosinophilic infiltration, respectively (11). This study examined the use of exhaled NO levels for the assess the levels of eosinophilic of allergic rhinitis in children. We present the following article in accordance with the TREND reporting checklist (available at http://dx.doi. org/10.21037/tp-20-314).

\section{Methods}

\section{Study design}

This study was a retrospective analysis and was designed to assess the application of $\mathrm{FeNO}$ and $\mathrm{nNO}$ levels for assess eosinophilic inflammation of allergic rhinitis. Symptom severity in children was assessed via questionnaire. Allergen detection, and blood tests present the degree of allergic rhinitis. FeNO and nNO levels were measured before and after treatment of rhinitis. We then analyzed the relevance of various indicators and the FeNO and nNO levels. In addition, we compared each of FeNO and nNO levels before and after treatment based on guideline and compared the degree of changes in the patients' symptoms via the questionnaire before and after the treatment. The study was conducted in accordance with the Declaration of Helsinki (as revised in 2013). The study was approved by the ethics committee from the First Affiliated Hospital of Guangzhou Medical University (GYFYY-2016-73) and individual consent for this retrospective analysis was waived.

\section{Participants}

The current study included children aged 3-12 years and diagnosed with allergic rhinitis from The First Affiliated Hospital of Guangzhou Medical University. In total, 42 males and 19 females were recruited from November 2019 to March 2020. All the children had been diagnosed with allergic rhinitis by an experienced clinician. The diagnostic criteria were based on standard treatment guidelines (12). Patients who were sensitive to at least one kind of allergen and who had allergic symptoms that lasted $>12$ months were included. Each child received treatment according to guideline. We excluded patients if: (I) they had concomitant asthma, rhinosinusitis, or nasal polyps; (II) they had cardiorespiratory-related diseases; (III) they had a respiratory infection before treatment; or if (IV) they previously underwent nasal surgery.

\section{Questionnaire administration}

The International Study of Asthma and Allergies in 
Childhood (ISAAC) is a useful tool for the assessment of allergic diseases (13). We designed our questionnaire based on this research. The questionnaire was divided into three sections: (I) basic personal information, including name, sex, age, and emergency contact number; (II) history of allergic rhinitis treatment and condition of family members with rhinitis; (III) frequency of symptoms, such as sneezing, rhinorrhea, nasal congestion, and itching of the nose or eyes, as well as the effects of symptoms on sleep, work, and activities of daily living. All levels of symptoms were divided into 10 scales. We considered each child's symptom score as the symptom sum score and the daily influence score as the daily activities sum scores. The parents or the children themselves completed the questionnaires independently.

\section{Allergen detection and blood test}

The number of eosinophils was measured via a blood test. The Phadia 1000 was used to detect allergen sensitization (Thermo Fisher Scientific Inc., USA), based on specific IgE and serum total $\mathrm{IgE}$ levels. $\mathrm{IgE}$ levels specific to allergens, including house dust mites, dog dander, egg whites, cow milk, wheat, shrimp, the German cockroach, and the Alternaria alter were measured.

\section{FeNO, nNO levels and Montelukast treatment}

During the measurement of FeNO levels, children used a filter to cover their mouths and expired into the device. During expiration, the children were asked to avoid inhaling and instead exhale gently for at least six seconds. There have three-year-old children we via moisture to measure his FeNO values based on the operation guide and other children were all measured by above method.

For detection of nNO, the children held a filter and blocked one nostril. The other nostril was open throughout the whole measurement. Each child was asked to inhale gas through the filter and then produce a whistle sound for at least ten seconds, without stopping.

Before the examination, we have excluded the children who have been used bronchodilators and other drugs that affect the airway caliber three months before examination. All children were asked not to exercise and fast for at least one hour, test in the same period and in a comfortable posture. Ensured there were no other environment factors may influence the measurement. During measurement of both FeNO and nNO, we made sure that the filter fit snuggly against the child's mouth or nostril without air leakage. The FeNO and nNO levels were measured using the Nano Coulomb Breath Analyzer (Sunvou-CA2122).

Every participant received personalized treatment under the diagnosis of clinician. Every patient had received the treatment with loratadine and lasts for three weeks. All the patients do not experience discomfort and completed the treatment and detect FeNO and nNO levels before and after treatment.

\section{Statistical analysis}

Data analysis was performed using the Statistical Package for the Social Sciences software 24.0 (SPSS, IBM Corp., Armonk, NY, USA). Normally distributed data are presented as mean \pm standard deviation. The Spearman models were used to evaluate whether a correlation existed between the FeNO and nNO levels and the other detection parameters. $\mathrm{P}$ values $<0.05$ were considered statistically significant. GraphPad Prism 8 was used to create the relationship diagram in our study.

\section{Results}

\section{We found no significant association between symptoms, as judged by the questionnaire, and $\mathrm{FeNO}$ and $\mathrm{nNO}$ levels}

This study focused on patients aged 3-12 years with allergic rhinitis. Sixty-one patient's characteristics, including symptom scores, and the effects of symptoms on each child's daily activities are summarized in Table 1. Sixty-one children with allergic rhinitis completed both the questionnaire and the FeNO and nNO tests. Both the FeNO levels and the symptoms sum scores were normally distributed as determined by the K-S normality test. We analyzed the correlation between symptom score and FeNO and $\mathrm{nNO}$ levels and found no significant correlation $(\mathrm{r}=0.028$, $\mathrm{P}=0.834 ; \mathrm{r}=-0.018, \mathrm{P}=0.893)$. Moreover, there have the positive correlation between $\mathrm{nNO}$ and the age $(\mathrm{r}=0.434$, $\mathrm{P}=0.001)$. All values of questionnaire, $\mathrm{FeNO}$ and $\mathrm{nNO}$ were tested and analyzed according to the guidelines (13-15). Reference value of FeNO: 5-25 ppb; nNO: 250-500 ppb.

\section{Association between FeNO and nNO levels}

The FeNO and nNO tests were used to detect the concentration of NO in the airway. FeNO and nNO levels can indicate inflammation at different locations. That is, the FeNO level reflects inflammation in the lower airway, and the nNO level indicates inflammation in the upper airway. 
Table 1 Patients' characteristics and rhinitis control scores

\begin{tabular}{|c|c|}
\hline Parameter & $\begin{array}{c}\text { Statistic } \\
(\text { mean } \pm S D)\end{array}$ \\
\hline Age (year) & $7.5 \pm 2.18$ \\
\hline Sneeze & $3.61 \pm 2.31$ \\
\hline Rhinorrhea & $3.72 \pm 2.28$ \\
\hline Nasal congestion & $3.71 \pm 2.49$ \\
\hline Nasal itching & $3.74 \pm 2.33$ \\
\hline Ocular itching & $2.34 \pm 2.31$ \\
\hline Conjunctival congestion & $1.05 \pm 1.89$ \\
\hline Watering eyes & $0.93 \pm 1.74$ \\
\hline Olfactory disorder & $0.85 \pm 1.69$ \\
\hline Eyelid edema & $0.98 \pm 1.75$ \\
\hline Symptoms sum scores & $20.93 \pm 10.68$ \\
\hline Interference with sleep & $2.98 \pm 2.75$ \\
\hline Impact on work or study & $2.51 \pm 2.26$ \\
\hline Influence on social activities & $1.84 \pm 2.08$ \\
\hline Impact on physical activities & $2.12 \pm 2.47$ \\
\hline Daily activities sum scores & $9.44 \pm 7.82$ \\
\hline Total-IgE (kU/L) & $366.46 \pm 515.30$ \\
\hline $\operatorname{EOS}\left(10^{9} / \mathrm{L}\right)$ & $0.40 \pm 0.29$ \\
\hline FeNO (ppb) & $16.31 \pm 9.14$ \\
\hline nNO (ppb) & $395.12 \pm 427.13$ \\
\hline FeNO after treatment(ppb) & $17.54 \pm 13.59$ \\
\hline $\mathrm{nNO}$ after treatment (ppb) & $420.82 \pm 261.36$ \\
\hline Symptoms sum scores after treatment & $11.92 \pm 10.68$ \\
\hline Daily activities sum scores after treatment & $4.51 \pm 6.56$ \\
\hline
\end{tabular}

We via the questionnaire from International Study of Asthma and Allergies in Childhood to measure the symptoms scores.

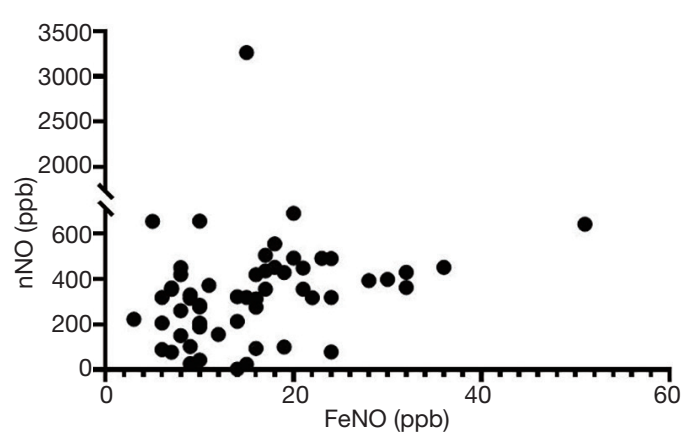

Figure 1 Correlation between FeNO and nNO levels. FeNO, fractional exhaled nitric oxide; nNO, nasal nitric oxide.
We observed a significant correlation between the FeNO and nNO levels $(\mathrm{P}<0.001)$ (Figure 1). However, when we compared the association between FeNO and nNO levels based on the EOS levels, which was $<0.3\left(10^{9} / \mathrm{L}\right)$, we found no significant correlation between the $\mathrm{FeNO}$ and $\mathrm{nNO}$ levels $(\mathrm{r}=0.241, \mathrm{P}=0.306)$.

\section{Correlation between various parameters and changes in $\mathrm{FeNO}$ and $n N O$ levels before and after the treatment of allergic rbinitis}

The EOS and total IgE levels are indices that are commonly used to diagnose allergic rhinitis in clinical settings. Both of $\mathrm{FeNO}$ and $\mathrm{nNO}$ values were strongly associated with total $\mathrm{IgE}$ (Figure $2 A$ ) and EOS (Figure $2 B$ ) values $(\mathrm{r}=0.414$, $\mathrm{P}=0.001 ; \mathrm{r}=0.293, \mathrm{P}=0.024$ and $\mathrm{r}=0.292, \mathrm{P}=0.025 ; \mathrm{r}=0.293$, $\mathrm{P}=0.025)$. Figure $2 C, D$ show the differences in $\mathrm{FeNO}$ and $\mathrm{nNO}$ levels before and after the treatment of children who completed the treatment. Via compared the symptoms sum scores and daily actives sum scores before and after the treatment from the questionnaire, we found that no matter symptoms sum scores, or daily activities sum scores were become lower after treatment. However, both of $\mathrm{FeNO}$ and $\mathrm{nNO}$ values after treatment were higher than before treatment. Besides, we tested FeNO and nNO for each patient who completed the treatment and compared whether the two detection indicators were relevant before and after treatment, respectively. Each of FeNO and nNO present significant correlation between the value of before and after treatment $(\mathrm{r}=0.612, \mathrm{P}<0.001 ; \mathrm{r}=0.601, \mathrm{P}<0.001)$. The values of sum scores afterment were present in Table 1 .

\section{FeNO or nNO level based on the different EOS levels}

Based on this result, we further evaluated the association between various parameters and FeNO and nNO levels at different EOS values. We divided patients in two group based on the cut line of $\operatorname{EOS}=0.3\left(10^{9} / \mathrm{L}\right)$. When the EOS level was $\geq 0.3\left(10^{9} / \mathrm{L}\right)$, the FeNO level was positively correlated with $\mathrm{nNO}$ levels $(\mathrm{r}=0.431, \mathrm{P}=0.006)$. However, the FeNO level was not correlated to any parameter when the EOS level was $<0.3\left(10^{9} / \mathrm{L}\right)$. The correlations between various parameters based on different levels of EOS were presented in Figure 3.

\section{Discussion}

The measurement of NO concentrations during expiration 

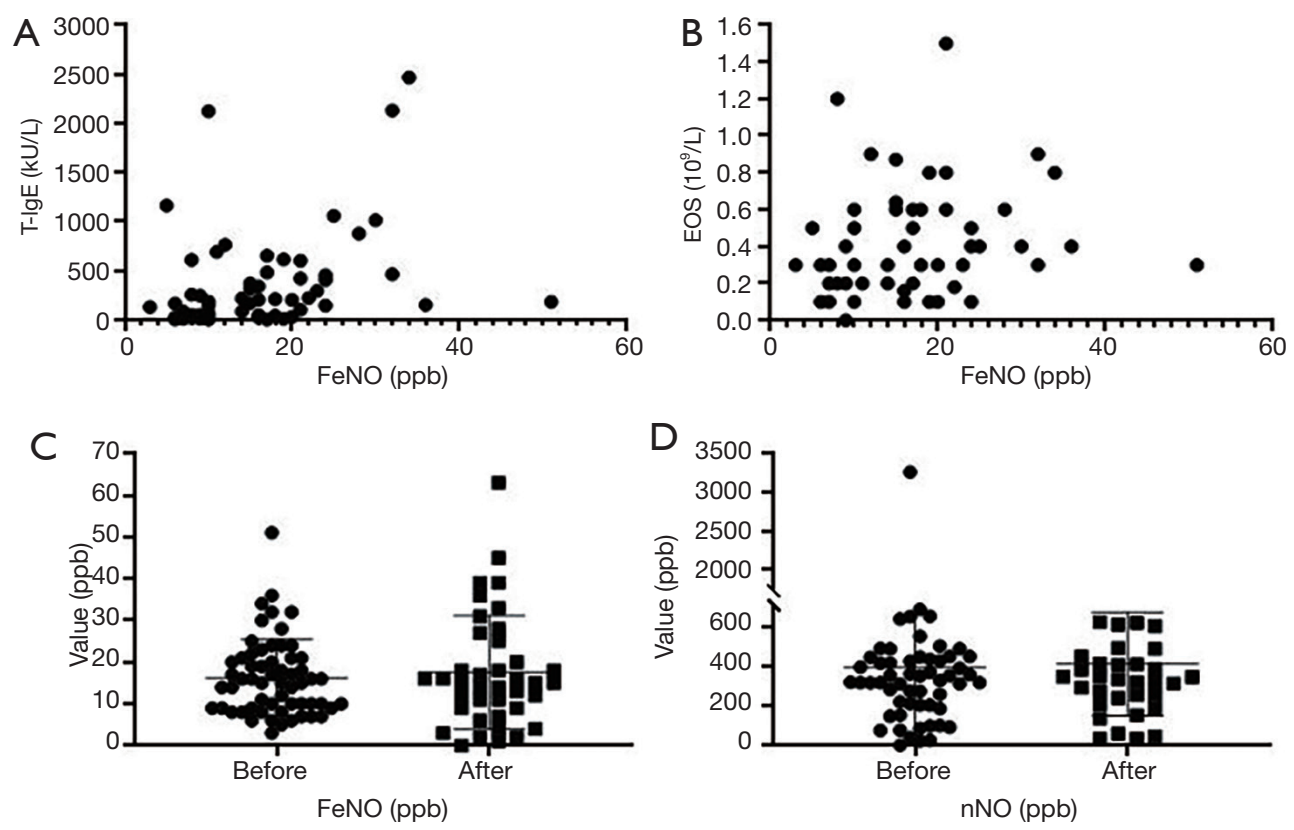

Figure 2 The relationship between FeNO and factors and the change of FeNO and nNO. Correlation between FeNO and (A) total IgE levels and (B) EOS values. The correlations between FeNO and nNO levels before and after treatment are indicated by (C) and (D). FeNO, fractional exhaled nitric oxide; total IgE, total immunoglobulin E; EOS, eosinophils nNO, nasal nitric oxide.

is not part of routine clinical practice. This may be because very few studies have examined its use, and there is no consensus as to its clinical measurement (16). Some factors, such as age and sex, can affect FeNO and nNO levels (17). In our study, the nNO level was positively associated with age. However, the relationship between FeNO level and age was not significant (FeNO level: $\mathrm{P}=0.164$; nNO level: $\mathrm{P}=0.001)$. This result was consistent with that of other studies $(3,17)$. In our analysis, no significant differences were observed between male and female participants. But some studies have produced different results. Interestingly, $\mathrm{FeNO}$ values were significantly higher in males compared to females $(10,18)$.

Assessments of symptom severity are commonly used in clinical settings. However, the symptom sum scores were not significantly correlated with $\mathrm{FeNO}$ and $\mathrm{nNO}$ levels $(\mathrm{P}=0.834 ; \mathrm{P}=0.893)$. These findings agreed with a previous study (19). That is, no association was observed between the nNO level and rhinitis symptoms because the manifestations of inflammatory infiltration do not present immediately. Therefore, FeNO or nNO levels are not reliable means of reflect the discomfort levels of allergic rhinitis.

The pathogenic mechanism of allergic rhinitis is airway inflammation with eosinophilic infiltration caused by the allergens. Because of TH2 lymphocytes and cytokines, eosinophils and mast cells invade the nasal mucosa (20). Therefore, the release of NO synthase from the epithelial cells produces a higher level of NO in the upper and lower respiratory tracts. Serum EOS and total IgE levels are useful indices for diagnosing allergic rhinitis. In summary, FeNO and nNO levels had a significant positive correlation $(\mathrm{r}=0.454, \mathrm{P}<0.001)$. Moreover, FeNO and level was positively correlated with EOS and total $\mathrm{IgE}$ levels $(\mathrm{r}=0.293, \mathrm{P}=0.024 ; \mathrm{r}=0.414, \mathrm{P}=0.001)$. But, when the data were classified according to EOS level, which was lower than $0.3\left(10^{9} / \mathrm{L}\right)$, the correlation will become lack of statistical significance. From this we can see, high concentration of NO can reflect airway inflammation. Thus, the concentration of NO can be a helpful indicate to reflect the high levels of eosinophils or total $\mathrm{IgE}$ and further assess the severity of allergic rhinitis. The reason could be because common allergens, including house dust, are small enough to reach the airways and cause higher levels of total $\operatorname{IgE}(21)$. Besides, we found out that total $\operatorname{IgE}$ can be one of the impact factors which make the level of $\mathrm{FeNO}$ and $\mathrm{nNO}$ change. A study out of China produced a similar result as our result (17). 


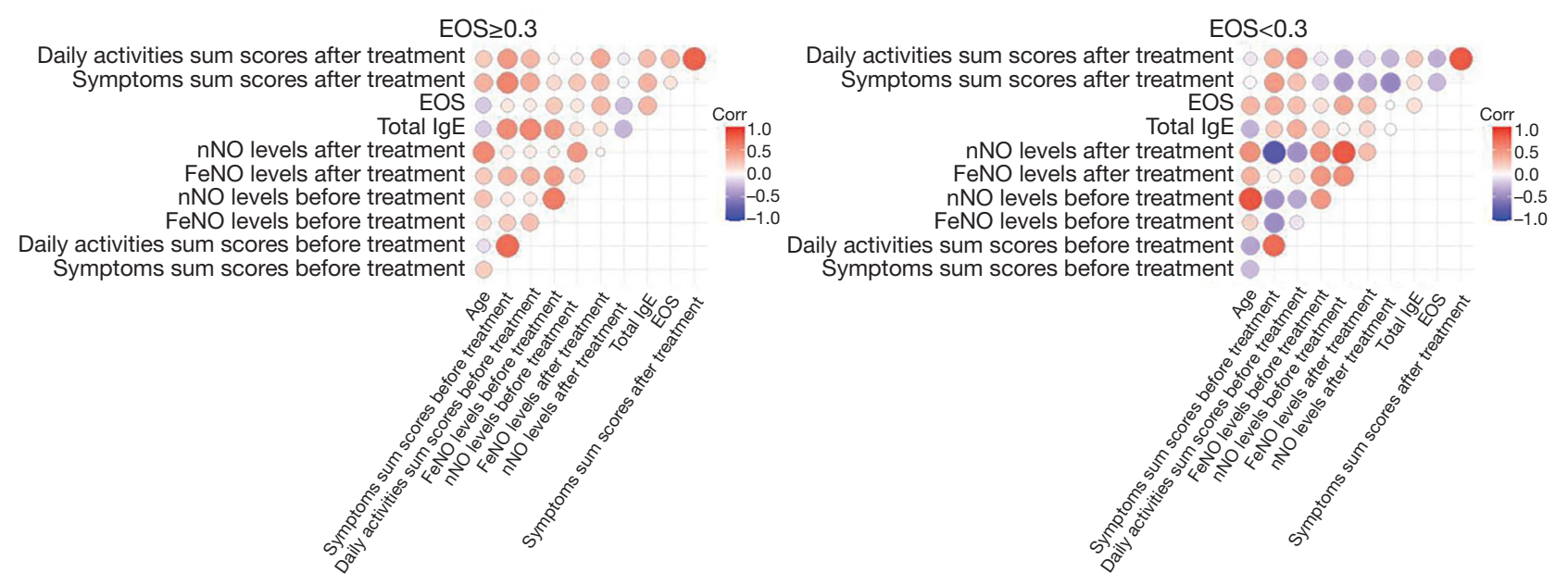

Figure 3 Correlation of various parameters based on different levels of EOS. FeNO, fractional exhaled nitric oxide; total IgE, total immunoglobulin E; EOS, eosinophils nNO, nasal nitric oxide.

Studies on use of nNO in clinical settings have emerged in recent years. nNO levels reflect the concentration of $\mathrm{NO}$ in the upper airways. However, there is no consensus regarding what levels of $\mathrm{nNO}$ can be used for clinical diagnosis (16). Several studies have used this method to compare $\mathrm{nNO}$ levels between patients with allergic rhinitis and healthy controls. They found that, in children with allergic rhinitis, nNO levels were significantly higher than those of healthy control children $(9,16,22)$. Based on this result, we sought to determine whether there was a direct association between $\mathrm{nNO}$ and $\mathrm{EOS}$ or total $\mathrm{IgE}$ levels. Interesting, we found the correlation was observed between $\mathrm{nNO}$ and both EOS and total $\mathrm{IgE}$, which result were similar than FeNO. $(\mathrm{r}=0.291, \mathrm{P}=0.025 ; \mathrm{r}=0.292, \mathrm{P}=0.025)$.

Several authors support the use of $\mathrm{FeNO}$ or $\mathrm{nNO}$ levels for measuring treatment effects in clinical settings, their result point out that both levels will decrease after treatment $(23,24)$. Nevertheless, when we compared FeNO and $\mathrm{nNO}$ values before and after treatment, we get different result. Our results showed that both FeNO and nNO levels were increase after treatment, even if the symptoms sum scores after treatment were significantly lower than before treatment via questionnaire. A review article from Europe summarize that FeNO levels do not decrease after treatment may have two possible reason. First, because the reduced diffusion capacity, anti-inflammatory drugs do not work efficiently. Second, airway remodeling may also reflect nNO levels do not decreased after treatment (25). Therefore, when evaluating the therapeutic effect by NO level, we tend to consider not only the change in FeNO and
nNO, but also combine other factor, such as the effects of NO production or airway remodeling.

Finally, there still have several limitations in our study. First, in view of that we only analysis 61 patients, the small sample size may limit our description of whether FeNO and $\mathrm{nNO}$ were related to total $\mathrm{IgE}$ and EOS, respectively. Second, due to each patient has a different performance of the treatment effect, we cannot integrally compare whether there has a significant change of the concentration of NO before and after the treatment.

\section{Conclusions}

In conclusion, both $\mathrm{FeNO}$ and nNO can reflect the levels of eosinophilic inflammation. When evaluating the effect of $\mathrm{FeNO}$ or $\mathrm{nNO}$, it is recommended to combine other influencing factors instead of simply comparing numerical changes. Moreover, the combined use of FeNO and $\mathrm{nNO}$ levels is a less invasive and potentially more tolerable and convenient method for the clinical assessment of the inflammation of allergic rhinitis.

\section{Acknowledgments}

Thanks to the assistance of clinical from Cheng-Zhi Zhao and all patients' cooperation in this research.

Funding: This work was supported by National Natural Science Foundation of China (Project No.: 81871736) and Guangzhou Science and Technology Foundation (Project No.: 201804020043). The funders had no role in study 
design, data collection and analysis, decision to publish, or preparation of the manuscript. No additional external funding was received for this study.

\section{Footnote}

Reporting Checklist: The authors have completed the TREND reporting checklist. Available at http://dx.doi. org/10.21037/tp-20-314

Data Sharing Statement: Available at http://dx.doi. org/10.21037/tp-20-314

Peer Review File: Available at http://dx.doi.org/10.21037/tp20-314

Conflicts of Interest: All authors have completed the ICMJE uniform disclosure form (available at http://dx.doi. org/10.21037/tp-20-314). The authors have no conflicts of interest to declare.

Ethical Statement: The authors are accountable for all aspects of the work in ensuring that questions related to the accuracy or integrity of any part of the work are appropriately investigated and resolved. The study was conducted in accordance with the Declaration of Helsinki (as revised in 2013). The study was approved by the ethics committee from the First Affiliated Hospital of Guangzhou Medical University (GYFYY-2016-73) and individual consent for this retrospective analysis was waived.

Open Access Statement: This is an Open Access article distributed in accordance with the Creative Commons Attribution-NonCommercial-NoDerivs 4.0 International License (CC BY-NC-ND 4.0), which permits the noncommercial replication and distribution of the article with the strict proviso that no changes or edits are made and the original work is properly cited (including links to both the formal publication through the relevant DOI and the license). See: https://creativecommons.org/licenses/by-nc-nd/4.0/.

\section{References}

1. Cheng L, Chen J, Fu Q, et al. Chinese Society of Allergy Guidelines for Diagnosis and Treatment of Allergic Rhinitis. Allergy Asthma Immunol Res 2018;10:300-53.

2. Wang XD, Zheng M, Lou HF, et al. An increased prevalence of self-reported allergic rhinitis in major Chinese cities from 2005 to 2011. Allergy 2016;71:1170-80.

3. Yoon J, Choi YJ, Lee E, et al. Allergic Rhinitis in Preschool Children and the Clinical Utility of FeNO. Allergy Asthma Immunol Res 2017;9:314-21.

4. Eguiluz-Gracia I, Mathioudakis AG, Bartel S, et al. The need for clean air: The way air pollution and climate change affect allergic rhinitis and asthma. Allergy 2020;75:2170-84.

5. Papadopoulos NG, Guibas GV. Rhinitis Subtypes, Endotypes, and Definitions. Immunol Allergy Clin North Am 2016;36:215-33.

6. Khan DA. Allergic rhinitis and asthma: epidemiology and common pathophysiology. Allergy Asthma Proc 2014;35:357-61.

7. Eguiluz-Gracia I, Pérez-Sánchez N, Bogas G, et al. How to Diagnose and Treat Local Allergic Rhinitis: A Challenge for Clinicians. J Clin Med 2019;8:1062.

8. Kirtsreesakul V, Leelapong J, Ruttanaphol S. Can a Pretreatment Visual Analog Scale Predict Treatment Outcome in Allergic Rhinitis? Int Arch Allergy Immunol 2020;181:285-91.

9. Chen QB, Chen L, Zheng XY, et al. The Clinical Significance of Nasal Nitric Oxide Concentrations in Preschool Children with Nasal Inflammatory Disease. Int Arch Allergy Immunol 2020;181:51-5.

10. Kim HB, Eckel SP, Kim JH, et al. Determinants and Clinical Application in Children With Allergic Airway Disease. Allergy Asthma Immunol Res 2016;8:12-21.

11. Duong-Quy S. Clinical Utility Of The Exhaled Nitric Oxide (NO) Measurement With Portable Devices In The Management Of Allergic Airway Inflammation And Asthma. J Asthma Allergy 2019;12:331-41.

12. Scadding GK, Kariyawasam HH, Scadding G, et al. BSACI guideline for the diagnosis and management of allergic and non-allergic rhinitis (Revised Edition 2017; First edition 2007). Clin Exp Allergy 2017;47:856-89.

13. Mata Fernández C, Fernández-Benítez M, Pérez Miranda $M$, et al. Validation of the Spanish version of the Phase III ISAAC questionnaire on asthma. J Investig Allergol Clin Immunol 2005;15:201-10.

14. Horváth I, Barnes PJ, Loukides S, et al. A European Respiratory Society technical standard: exhaled biomarkers in lung disease. Eur Respir J 2017;49:1600965.

15. American Thoracic Society; European Respiratory Society. ATS/ERS recommendations for standardized procedures for the online and offline measurement of exhaled lower respiratory nitric oxide and nasal nitric oxide, 2005. Am J Respir Crit Care Med 2005;171:912-30. 
16. Wen YS, Lin CY, Yang KD, et al. Nasal nitric oxide is a useful biomarker for acute unilateral maxillary sinusitis in pediatric allergic rhinitis: A prospective observational cohort study. World Allergy Organ J 2019;12:100027.

17. Zhu Z, Xia S, Chen X, et al. Factors associated with exhaled nitric oxide in children with asthma and allergic rhinitis. Clin Respir J 2020;14:9-15.

18. Olivieri M, Talamini G, Corradi M, et al. Reference values for exhaled nitric oxide (reveno) study. Respir Res 2006;7:94.

19. Olin AC, Hellgren J, Karlsson G, et al. Nasal nitric oxide and its relationship to nasal symptoms, smoking and nasal nitrate. Rhinology. 1998;36:117-21.

20. Kakli HA, Riley TD. Allergic Rhinitis. Prim Care 2016;43:465-75.

21. Hara J, Fujimura M, Myou S, et al. Eosinophilic inflammation, remodeling of lower airway, bronchial

Cite this article as: Luo JY, Chen HA, Ma J, Xiao YX, Yao JJ, Liang JM, Du YS, Wang F, Sun BQ. Clinical application of fractional exhaled nitric oxide and nasal nitric oxide levels for the assess eosinophilic inflammation of allergic rhinitis among children. Transl Pediatr 2021;10(4):746-753. doi: 10.21037/ tp-20-314 responsiveness and cough reflex sensitivity in nonasthmatic subjects with nasal allergy. Int Arch Allergy Immunol 2006;140:327-33.

22. Wang PP, Wang GX, Ge WT, et al. Nasal nitric oxide in allergic rhinitis in children and its relationship to severity and treatment. Allergy Asthma Clin Immunol 2017;13:20.

23. Wu M, Wang Q, Zhang K, et al. The effect of nasal irrigation in the treatment of allergic rhinitis. Lin Chung Er Bi Yan Hou Tou Jing Wai Ke Za Zhi 2014;28:287-9.

24. Vo-Thi-Kim A, Van-Quang T, Nguyen-Thanh B, et al. The effect of medical treatment on nasal exhaled nitric oxide (NO) in patients with persistent allergic rhinitis: A randomized control study. Adv Med Sci 2020;65:182-8.

25. Lehtimäki L, Karvonen T, Högman M. Clinical Values of Nitric Oxide Parameters from the Respiratory System. Curr Med Chem 2020;27:7189-99. 